\title{
POIESIS DEL TIEMPO Y DEL MOVIMIENTO: UNA NUEVA MIRADA A LA ONTOLOGÍA ARISTOTÉLICA
}

\author{
Diana María Acevedo Zapata* \\ doi:10.11144/Javeriana.uph31-63.ptma
}

\begin{abstract}
RESUMEN
Acudo al concepto de poiesis (producción), a partir del uso que hace de este Paul Valéry, para considerar el tiempo y el movimiento como conceptos producidos en el marco de un proyecto de comprensión del mundo natural. Bajo la idea que la filosofía es una manera de construir la inteligibilidad de los fenómenos a partir de conceptos, intento mostrar la coherencia entre la construcción del concepto de tiempo y la del concepto de movimiento.
\end{abstract}

Palabras clave: producción; filosofía de la naturaleza; teleología; actualización; potencialidad

\footnotetext{
*Universidad Nacional de Colombia, Bogotá, Colombia.

Recibido: 02.09.13 ACEPTADO: 20.03.14 Disponible EN líNeA: 02.12.14

Una versión en borrador del texto fue presentada en el II $^{\circ}$ Congreso Nacional de Filosofía realizado en Concepción, Chile, del 15 al 18 de noviembre de 2011. La culminación de este trabajo se dio gracias al apoyo de la beca Orlando Fals Borda I-2012, de la Facultad de Ciencias Humanas, Universidad Nacional de Colombia. Agradezco además la discusión del mismo en el Núcleo de investigación en Estética, línea de Poética y Ontología, de la Universidad Javeriana de Bogotá.

Para citar este artículo: Acevedo Zapata, D.M. (2014). Poiesis del tiempo y del movimiento: Una nueva mirada a la ontología aristotélica. Universitas Philosophica, 31(63), pp. 267286, ISSN 0120-5323, ISSN en línea 2346-2426, doi: 10.11144/Javeriana.uph31-63.ptma
} 


\title{
POIESIS OF TIME AND MOVEMENT: A NEW PERSPECTIVE ON THE ARISTOTELIAN ONTOLOGY
}

\author{
Diana María AceVedo Zapata
}

\begin{abstract}
Having in mind the concept of poiesis (production), as Paul Valéry uses it, time and movement are presented as concepts produced within the project of understanding the natural world. From the idea of philosophy as a way of constructing through concepts the intelligibility of phenomena, I will show the coherence between the construction of the concept of time and that of the concept of movement.
\end{abstract}

Key words: production; philosophy of nature; teleology; actualization; potentiality 
PARA hablar De la PRODUCCiÓN, DE POIESIS, resulta necesario precisar su sentido y su uso en un contexto muy particular: la pregunta por el tiempo. Este contexto se define todavía más cuando me ubico en una pregunta sobre el tiempo y, por consiguiente, en un modo de respuesta; me refiero a la perspectiva aristotélica. Para entender la razón de semejante unión tiempo, Aristóteles, producción- empiezo la reflexión precisando un modo de comprender la producción.

Es conocida la distinción aristotélica entre las cosas naturales, que tienen en sí mismas el principio de cambio, y los productos de la técnica, que tienen en otro su principio de cambio (Met. 1070a7; Fís. 192b21). Esta diferencia propuesta por Aristóteles entre naturaleza y técnica ha determinado en gran medida lo que solemos entender por producción; esta se conecta etimológicamente con la poiesis que encontramos inscrita, dentro de la clasificación de los saberes, en la techne, lejos de la physis (Met. 1025b25). Me interesa plantear una forma de producción humana que sirva de base a mi interpretación del concepto de tiempo aristotélico como algo producido conceptualmente. La tesis central es que el tiempo, si bien Aristóteles lo inscribe en la physis, es un concepto construido por él, y por tanto su construcción es humana, en oposición a la producción de la naturaleza. Por lo anterior, la filosofía de la producción de Paul Valéry me resulta útil en cuanto recurre justamente a la distinción entre dos modos de producción: la formación natural y la construcción humana ${ }^{1}$. Esta distinción es conquistada por Valéry a través de un bello ejercicio fenomenológico sobre un objeto ambiguo: una concha. La ambigüedad consiste en que la concha parece hecha, por lo que sugiere la posibilidad de construcción al modo humano. Sin embargo, el proceso de formación que le da lugar es de un carácter diferente: "vivido y no realizado" (Valéry, 1993, p. 155). La diferencia entre el modo de producción que Valéry llamaría propiamente humano (construcción) y aquel propiamente natural (formación) me permite precisar las particularidades del proceso de construcción del concepto de tiempo aristotélico. Por eso me resulta útil el vínculo que Valéry establece entre hacer y conocer: pues no encuentra "otra medida para el conocimiento

\footnotetext{
${ }^{1}$ La discusión que sirve de marco a este problema es más amplia: dentro de las cosas que llegan a ser ¿cómo hacer inteligible este llegar a ser? Valéry busca resaltar el proceso por el cual algo llega a ser y no el resultado del mismo: "Puede que nos veamos arrastrados a considerar con mayor complacencia e incluso con mayor pasión, la acción que hace, que la cosa hecha" (Valéry, 1998, p. 109).
} 
que el poder real que confiere. Sólo sé lo que sé hacer" (Valéry, 1993, p. 153). En suma, el hacer humano, es decir, la construcción, es un modo de hacer inteligible o de conocer.

Esta visión valeriana de la construcción me permite transferir la discusión al ámbito conceptual para considerar el tiempo desde la perspectiva de la construcción (no formación) del concepto. Este proceso de construcción consiste en el modo de hacer inteligible el fenómeno. Así, podemos conocer en la medida en que podemos construir conceptos. La construcción es, pues, el sentido de producción que me interesa para examinar el concepto de tiempo como una poiesis interior a la ontología aristotélica. Aristóteles le da orden e inteligibilidad al fenómeno del tiempo, distribuyendo sus partes y la relación entre ellas. La particularidad de esta poiesis, en su sentido propio de construcción, es la siguiente: el tiempo se hace dependiente del movimiento, y este último se hace inteligible privilegiando cortes inmóviles o estados detenidos $^{2}$ que se determinan como más perfectos [teleion] y capaces de explicar teleológicamente el movimiento. Esta particular composición del movimiento a partir de cortes inmóviles determina la construcción del tiempo como un compuesto de unidades y límites. La tesis que defiendo toma distancia de un elemento crucial en la definición aristotélica del tiempo: su carácter natural y cosmológico. Me alejo de una interpretación que asigne al tiempo la condición de fenómeno natural para asumirlo como una construcción humana en sentido valeriano. Para llevar a cabo mi propósito, primero desarrollaré la diferencia entre construcción y formación, luego me detendré en la producción aristotélica de los conceptos tiempo y movimiento.

\section{Producción humana y producción natural}

Un OBJETO DE PRONTO IRRUMPE EL "DESORDEN ordinario del conjunto de las cosas sensibles" (Valéry, 1993, p. 139), se hace notable, privilegiado, en medio de lo que es indiferente. Aparece la concha entre las cosas naturales y se destaca por su forma. Este énfasis en la forma viene acompañado por un ojo que la ve, por una expectativa que se colma. Pues no parece haber

\footnotetext{
2 Poses en términos deleuzianos: "El movimiento [en la antigüedad] no hace más que expresar una «dialéctica» de las formas, una síntesis ideal que le da orden y medida. El movimiento así concebido será, pues, el paso regulado de una forma a otra, es decir, un orden de las poses o de los instantes privilegiados" (Deleuze, 1984, p. 17).
} 
formas notables por sí mismas, incluso tal vez no hay formas por sí mismas. Más bien, estas se hacen notables para alguien que encuentra en el medio natural cosas que parecen hechas de un modo semejante a como él las hace. Llamamos Naturaleza, la Producente, a quien "encargamos de producir todo aquello que no sabemos hacer y que, sin embargo, nos parece hecho (...) todo lo demás se lo ofrecemos al «azar»" (Valéry, 1993, p. 151). Hay aquí una opción de Valéry por cierto modo de hacer como lo más inteligible y claro para nosotros: la técnica. El objeto ambiguo trae consigo la inquietud de su semejanza con las cosas que hacemos técnicamente y el misterio de su proceso de formación, de las operaciones que lo conducen.

Esta concha que sostengo y giro entre mis dedos, y que me ofrece un desarrollo combinado de los temas sencillos de la hélice y la espiral, me introduce a su vez en un asombro y una atención que producen lo que pueden: observaciones y precisiones exteriores, ingenuas preguntas, comparaciones «poéticas», imprudentes «teorías» en estado naciente... (Valéry, 1993, p. 140)

El primer intento de Valéry ante el objeto ambiguo es la descripción del geómetra. No me interesa desarrollarlo en extenso, tan sólo resulta relevante mencionar que se refiere al hecho de que creemos saber de la concha, creemos entenderla a través de definiciones y conceptos, lo cual resulta un proceso exterior que no puede abarcar la totalidad de la concha: siempre se nos escapa su espontaneidad y diversidad. A este modo exterior de saber se opone la divisa metodológica de la tabula rasa, una especie de epoché que le permite a Valéry dejar de lado por un momento las cosas que cree saber.

Planteo por lo tanto mi pregunta ingenua (...) Miro por primera vez esta cosa encontrada; constato lo que he dicho, al tocar su forma, me siento confundido. Es entonces cuando me pregunto: ¿Quién ha hecho esto? (...) Mi primer movimiento de la mente ha sido pensar en el Hacer. (Valéry, 1993, p. 144)

Este es el énfasis que me interesa: el hacer como aquello humano por excelencia. He entendido algo cuando lo he hecho, y por eso el nivel del hacer se extiende hasta el pensamiento. Entonces, "«Explicar» no es otra cosa que describir una manera de Hacer: no es más que rehacer con el pensamiento" (Valéry, 1993, p. 145). Es claro que lo anterior implica poner un límite a lo que podemos saber, que nos recuerda incluso la posición 
restrictiva del Wittgenstein del Tractatus ${ }^{3}$. Propongo considerar este límite desde las posibilidades que abre al pensamiento y no desde lo que recorta o cierra. Aunque no podamos llegar a explicar la formación natural, dado que no podemos hacerla a voluntad, resulta muy notable considerar las construcciones del pensamiento: construcciones que pretenden responder el porqué de los fenómenos, desde el punto de vista del cómo. Lo anterior implica una mirada consciente de que todo pensar es hacer con el pensamiento, y con ello, me gustaría agregar, es un hacer a través de conceptos. Aun cuando dicho hacer cruza el límite de lo que puede hacer e imagina mecanismos de operación que no le son accesibles, resulta interesante reflexionar sobre su modo de construcción, sobre las necesidades ante las cuales responde y sobre las preguntas y artificios que enfrenta. La verdad de una teoría o una construcción conceptual no juega aquí un papel central. De hecho, el mismo Valéry sostiene: "si la hipótesis es seductora, si la teoría es hermosa, yo disfruto sin pensar en lo verdadero" (Valéry, 1993, p. 153).

Entonces, la concha permite clarificar en qué consiste el hacer humano en oposición al natural, lo cual resulta muy notable si se habla de lo que podemos conocer los seres humanos. Supongo, a modo de hipótesis, que puedo hacer la concha, que puedo fabricarla, dado que su forma definida y ordenada parece hecha por alguien. Justo en este punto aparece una distinción clave: tengo que escoger una materia para imprimir, modelar y cincelar la figura de la concha. En la producción humana la acción, la materia y la forma están disociadas y no son necesarias, debo escogerlas yo como productora. Así me es posible determinar una materia que nunca será necesaria, ni estará determinada necesariamente por la forma. Escojo también una forma, un objetivo e incluso cuánto tiempo le dedico a mi trabajo de producción. Esta amplitud de elecciones -materia, tiempo y figura-, que requieren la atención de mi conciencia, caracterizan para Valéry el hacer humano ${ }^{4}$. El molusco que produce la concha está en una situación radicalmente diferente, "se trata de una condición absoluta: si no se puede hacer más que una cosa, y de una sola manera, se hace como por sí misma; y por lo tanto dicha acción

\footnotetext{
${ }^{3}$ Para un desarrollo en extenso de esta idea, véase: Brigante, 2008, capítulo 1.

${ }^{4}$ Creo que aquí es pertinente restringir el alcance explicativo de esta forma de exponer la acción y el conocimiento humanos: a mi juicio, Valéry resulta muy útil cuando se trata de un autor como Aristóteles, pero no quiero decir con esto que todo modo de hacer con el pensamiento deba recurrir a estos mismos elementos.
} 
no es verdaderamente humana (porque el pensamiento no es necesario), y nosotros no la comprendemos" (Valéry, 1993, p. 148). Nosotros mismos como seres humanos estamos compuestos de procesos a los cuales tenemos acceso, tales como respirar, digerir, etc., pero que no podemos conocer, ni discernir sus operaciones superpuestas y mezcladas, indistintas. De hecho, no necesitamos conocer dichos procesos para que se efectúen. Por el contrario, la producción humana se presenta completamente discernible; la forma, la acción, la materia y el tiempo hacen parte de la acción que se da paso a paso, ya que son operaciones numerables y definidas. El hecho de poder interrumpir la producción humana es muy notable en la medida en que el momento de la terminación de la obra está sujeto a un juicio exterior a la misma, bien sea porque así se decide, bien sea por falta de atención, es decir que puedo detener la producción puesto que mi vida no está implicada en ella. Finalmente, una última precisión: la producción humana suele estar determinada por la utilidad, pero en el caso del arte "nada vital lo exige, nada vital lo prescribe. No procede de una necesidad, que además, lo determinaría por entero, y aún menos podríamos atribuirlo al azar" (Valéry, 1993, p. 151). La utilidad resulta así un criterio importante para determinar el carácter de la producción humana, pues el arte se distancia de cierta técnica que está supeditada a las necesidades prácticas.

Aristóteles distingue entre los procesos de la técnica y aquellos que ocurren por naturaleza, como se mencionó antes. Los elementos que permiten hacer inteligibles ambos procesos (materia, forma, finalidad...) son comunes a ambos, aunque también permiten marcar la diferencia, tal como tener un principio interno o externo del movimiento. Por su parte, el tiempo se inscribe en los estudios naturales y, aunque no se puede decir que es una de las cosas que son por naturaleza o que tienen en sí mismas el principio de movimiento y de reposo, es algo inherente a la naturaleza. De esta manera, el tratado sobre el tiempo nada tiene que ver con el desarrollo de una técnica, ni con un producto de la misma. Por eso la introducción de la diferencia entre formación y construcción me permite afirmar que el tiempo no sólo no es una cosa, sino que tampoco pertenece al ámbito de lo natural, no es inherente a la naturaleza, como la construcción de la concha no es natural respecto del artesano. Busco darle un giro al problema del tiempo al considerarlo como una producción conceptual, y al distinguir sus condiciones de producción como específicamente humanas, para ello recurro a Valéry. Así espero mostrar cómo el concepto de tiempo se construye a través de la determinación de sus partes y de la relación que mantienen entre ellas 
y con otro concepto fundamental para Aristóteles, a saber, el movimiento. La conexión entre movimiento, número, antes y después, ahora, unidad y límite, elementos con los Aristóteles compone el concepto de tiempo, no es necesaria en la medida en que es posible pensarla de otra manera. A esto apunta la noción de construcción. De modo que me interesa dilucidar las razones que, desde los presupuestos de la ontología aristotélica, conducen a este y no a otro concepto de tiempo. Sin embargo esta aproximación, que desnaturaliza el tiempo aristotélico con herramientas tomadas de la poiética de Paul Valéry, es una opción de interpretación que como tal no pretende excluir otros caminos posibles. Me refiero con esto a que el primado de la conciencia y la capacidad de elección, así como la dualidad entre forma y materia, en la perspectiva de Valéry, son modos de explicar la acción que resultan útiles y encajan con la ontología aristotélica. Así pues, se trata de un ensamble particular y no de una teoría general de la acción, válida para toda construcción conceptual o todo proceso posible. Si bien el límite entre naturaleza y técnica, o entre naturaleza y arte, es claro y definitivo para Valéry, a mi juicio dicho límite permite explicar ciertos modos de producción, pero no todos. Con estas observaciones doy paso al desarrollo concreto de lo que me permito llamar el proceso de construcción del concepto de tiempo en Aristóteles.

\section{Una doble producción aristotélica}

\subsection{Movimiento}

EL TIEMPO Y EL MOVIMIENTO PRETENDEN INSCRIBIRSE dentro de los asuntos físicos, como fenómenos necesariamente atados a las cosas que tienen en sí mismas el principio de cambio ${ }^{5}$ o por decirlo en términos valerianos, las cosas que se forman a sí mismas, como la concha del molusco. El problema

\footnotetext{
${ }^{5}$ Aristóteles en muchas ocasiones hace un uso indistinto de los términos movimiento [kinesis] y cambio [metabolē], pero su distinción tiene un uso técnico muy particular en la clasificación de las cosas que cambian (Fis. 225a1-6; 225a34-225b2). En lo que sigue haré uso indistinto de estos términos, al igual que el autor, salvo cuando sea necesario atenerse al tecnicismo. En rigor, el concepto de cambio incluye la generación y la corrupción (Fís. 225a1-6); sin embargo, Aristóteles no desarrolla la particularidad de estos cambios dentro la definición del movimiento. Esto se puede notar en 218b18: "de momento no hay ninguna diferencia para nosotros entre decir movimiento y cambio".
} 
es que esta inscripción del tiempo y el movimiento en las cosas físicas no es un punto de partida simple, sino que, por el contrario, requiere una serie de presupuestos que no están discutidos como tales en la Física. El primero de ellos es la opción por las cosas en movimiento como lugares de explicación del movimiento, del proceso. Esta decisión, ya sea implícita o explícita, es un modo de dar orden a un fluir que se consideraría en primera instancia desordenado e ininteligible. La atención de Aristóteles se concentra en el cosmos cambiante, esta es la materia a la cual quiere dar orden y forma, inteligibilidad. El conocimiento es conocimiento de causas (Fís. 184a14-15), de porqués, pero si seguimos la línea de Valéry, conocer la formación de la concha $-\mathrm{o}$ de aquello que queremos conocer- implicaría poder hacerla, poder observar su proceso de producción. Así, el principio de causalidad sería una forma de imaginar "de sustituir nuestras lagunas por nuestras combinaciones" (Valéry, 1993, p. 153). Nuestras lagunas son aquellas cosas que no podemos hacer, en vista de ello, recomponemos, combinamos, lo que sí podemos hacer y lo sustituimos: "la pregunta es humana, la respuesta demasiado humana" (Valéry, 1993, p. 153). Aristóteles quiere explicar el movimiento y para ello lo recompone a través de lo que considera más inteligible: las cosas estáticas, los cortes inmóviles. Escoge estados que considera acabados o completos y los determina como el principio explicativo del movimiento, los privilegia sobre el fondo indefinido de los procesos. Compone el movimiento teleológicamente, organiza sus partes y construye un conjunto cerrado. Esta es la primera composición de Aristóteles.

El primer paso consiste en discernir un estado inicial y uno final entre los cuales pueda ocurrir el proceso o movimiento: "Lo que está en movimiento se mueve desde algo hacia algo [to kinoumenon kineitai ek tinos eis ti]" (Fís. 219a11). Pero la cosa cuando cambia no puede estar ni en el estado inicial, ni en el estado final; el movimiento ocurre entre estos y eso significa que se opone a los estados acabados [teleion], es inacabado [ateles]. Pero ¿cómo distinguir el estado inicial y el final? Se trata de poner un punto al movimiento, ya sea inicial o final, de marcar un indivisible, una unidad estática entre el continuo divisible. En este punto interviene el fin [telos], aquello hacia lo cual [hou heneka] se mueven las cosas, para indicar el punto final por excelencia a través del cual se puede explicar un movimiento ${ }^{6}$. Esta

\footnotetext{
${ }^{6}$ M. R. Johnson (2005) nos recuerda que la teleología está a la base de la filosofía aristotélica, particularmente a través de su idea de causalidad. Esto significa que la explicación de las
} 
elección del fin resulta ser la primera operación ordenadora que construye, sobre la materia amorfa y desordenada del cosmos cambiante, un edificio de conceptos, un engranaje: le doy forma al mundo cuando privilegio ciertos instantes suyos y hago de ellos la razón de ser de los demás. Este modo de componer el movimiento está justificado a través de una analogía, o en otras palabras, un modo de combinar y sustituir elementos menos inteligibles por elementos más inteligibles. Me refiero a la línea y el punto: el primero, permite entender el movimiento como un continuo que se da entre los estados acabados, el segundo, dichos cortes inmóviles o estados acabados hacia los cuales tienden los movimientos.

La línea es el continuo por excelencia, pero hay que notar que su continuidad descansa en la función divisora de los puntos: un punto es el límite de dos segmentos de la línea. Lo anterior no implica que la línea esté compuesta de puntos, pues estos como tales no existen más que en su función de límites indivisibles, y por tanto ellos mismos no tienen longitud y tampoco partes. Por ello es imposible que dos puntos se toquen, puesto que dependen como límites de aquello que limitan, es decir, su función consiste en mantener separados dos segmentos; suponerlos en contacto implica en último término que no haya segmentos. Pero el contacto de los mismos resulta imposible, no sólo porque anula toda posibilidad de longitud, sino además porque, dado el carácter indivisible de los puntos, ¿cómo pueden tocarse si no tienen límites propiamente para entrar en contacto? (Fís. 231b 3-5). En el caso del movimiento, el segmento corresponde precisamente al movimiento, en tanto opuesto a los estados acabados, puntos, que lo limitan y determinan su comienzo y final. Así, el movimiento es a los estados acabados como la longitud es a los puntos indivisibles. Aristóteles ilustra esto a través de un ejemplo (Fís. 231b19-232a10): hay una distancia (entiéndase magnitud o tramo $)^{7} \mathrm{ABC}$, recorrida por un móvil $\mathrm{X}$ con el movimiento

cosas incluye la determinación de aquello para lo cual algo es o existe, y esto hace parte de una explicación causal. En el caso del movimiento, si vamos más lejos, encontramos que a esta prioridad del fin, en conjunción con la importancia de la definición y la forma, le corresponde la prioridad de las cosas substantes por encima de los cambios o movimientos. Estos solo pueden ser explicados con el auxilio de la forma acabada hacia la cual cambia o tiende una cosa en cuanto hace parte de una clase o categoría de cosas.

${ }^{7}$ Düring (2000, p. 501) sugiere una traducción bastante particular del término megethos, cuya habitual traducción es magnitud, y prefiere entenderlo como el trecho por recorrer. Así propone entender la magnitud como distancia. Esto resulta plausible en la medida en que 
LMN. Es imposible que $\mathrm{X}$ recorra LMN a través de tramos indivisibles L, M y N. En ese caso, el movimiento estaría compuesto de inmovilidad dado que $\mathrm{X}$ estaría en reposo al pasar por A y culminar allí un movimiento, luego en B y, asimismo, en C. De este modo, resulta necesario establecer un intermedio entre un estado y otro. La clave es que, si bien el movimiento no está compuesto de indivisibles, se delimita a través del privilegio de estados acabados que son indivisibles, en tanto implican reposo y le dan sentido y culminación a todo el proceso.

Ahora bien, hay que notar que el ejemplo anterior está fundado en el modelo del movimiento local, donde se encuentran: un recorrido o trecho, un móvil con su movimiento sobre este recorrido y el tiempo que toma el mismo. Pero ¿se puede establecer una analogía con los demás tipos de movimiento a este respecto? ${ }^{8}$ Para ello Aristóteles distingue tres cosas respecto de todos los movimientos: lo que [ho], en lo que [en hoi] y cuándo [hote]. Como se puede notar, en los demás casos de movimiento se mantiene la misma relación establecida en el ejemplo, a saber, tramo, móvil y movimiento. Con la salvedad de que aquí, aquello en lo que se da el movimiento puede ser, además de un lugar, una afección -la única condición de esto es que aquello en lo que se desarrolla la afección sea unitario y divisible-. Entonces, si bien podemos decir que a cada tramo de un recorrido le corresponde una parte del movimiento, en el caso de una afección, pese a que no se está dividiendo una porción de espacio determinada posicionalmente, puede también ser divida en momentos del movimiento y las partes de aquello sobre lo cual se da el movimiento?

el movimiento local es el modelo de movimiento que se usa en la Física para comprender las demás modalidades de movimiento, ya que es el más común y cognoscible para nosotros (Fis. 208a31-32). Sin embargo, lo anterior no implica que las conclusiones queden restringidas a dicho movimiento, como veremos enseguida.

${ }^{8}$ Coope $(2005$, p. 53) duda de la posibilidad de esta analogía a través del ejemplo de un violín, si bien parece necesario que en un movimiento espacial, siempre el espacio entre un estado y otro deba ser llenado. No es claro que esto suceda en el paso de un sonido entre un tono y otro, pues ¿sobre qué ocurre el movimiento del sonido? Considero que esta objeción se supera por los argumentos que siguen.

${ }^{9}$ En el ejemplo del violín, Coope quiere establecer el modelo local en los mismos términos para una afección como el sonido. Pero el punto es que en el caso del sonido, aquello en lo que se da el cambio no es distancia. De allí que su análogo sería más bien el medio en que se da el sonido, que no está determinado posicionalmente. Por eso es necesario agregar que la distinción tiene que ser modal, de tal manera que se vea que la diferencia entre la 
Considero que este es un ejemplo claro de hacer con el pensamiento, en el que Aristóteles recompone o rehace el movimiento para poder explicarlo. Pero para rehacerlo tuvo que tomar decisiones y privilegiar ciertos elementos. Así, el movimiento se reduce a la composición de los momentos del continuo LMN, y X como el móvil que permanece durante el cambio. El movimiento se divide y se determinan sus partes: un punto inicial, un intermedio y un punto final. Estos elementos que toma de la construcción de una línea configuran la forma de la continuidad: Aristóteles no acepta la ruptura, los saltos, como un modo de explicación del movimiento, pues le parece imposible reconstruir el movimiento a partir de la inmovilidad. Los puntos que marcan dos segmentos como diferentes, al mismo tiempo que mantienen unidos los segmentos, divisibles al infinito, son discontinuos entre sí, pues los separa la línea. Hay una diferencia de naturaleza entre los puntos indivisibles y el segmento divisible. Aristóteles pone toda la carga explicativa al nivel de los puntos indivisibles, es decir, al nivel de los estados inmóviles. Esto es el movimiento: el compuesto particular que surge de privilegiar ciertos estados discontinuos y estáticos, determinarlos como más perfectos, acabados o culminantes, y componerlos en un orden de sucesión sobre el fondo continuo de la movilidad -sobre la sucesión aparecerá el tiempo como veremos adelante-. ¿Cómo escoge Aristóteles los puntos culminantes del movimiento en virtud de los cuales este se hace inteligible? Diríamos que, en términos de Valéry, del modo más humano posible. Consideremos el procedimiento aristotélico para privilegiar unos estados sobre otros.

Aristóteles define el movimiento a partir de la distinción entre dynamis y entelecheia ${ }^{10}$ para indicar su carácter inacabado: "Puesto que distinguimos en cada género lo actual y lo potencial, el movimiento es la actualidad [entelecheia] de lo potencial [dynamei ontos] en cuanto a tal [he tou dynamei

estructura de los tipos de movimientos no afecta el concepto mismo de movimiento, sino una modalización del mismo.

${ }^{10}$ El término usado para actualidad no siempre es entelecheia, además Aristóteles usa energeia. Propiamente, el primer término no existe en griego, es compuesto por Aristóteles a partir de los vocablos en-telos-echein, para denotar tener dentro el fin; energeia por su parte, significa estar en acción. No obstante, Aristóteles los usa indistintamente como lo atestigua el presente parágrafo, donde el problema inicial es planteado en los términos del primero y la distinción se hace en los del segundo. Algunos traductores, como García Yebra, marcan la diferencia usando el término 'acto' para energeia y 'actualidad' para entelecheia. En adelante haré uso indistinto de los términos actualidad y acto. 
ontos entelecheia, hei toiouton kinesis estin]" (Fís. 201a10). Dado que el movimiento se postula como inseparable de las cosas que se mueven, es decir, de las cosas concretas, de las substancias, este resulta afectado por el modo como se predica ser de ellas (Fís. 200b33). Potencia y acto hacen parte de los sentidos en los que se predica el verbo ser $\mathrm{y}$, como tales, son distinguibles dentro de cada género de $\operatorname{cosas}^{11}$. La definición del movimiento está ligada a esta distinción, en la medida en que permite poner en relación, digamos componer, dos elementos: por un lado, los estados acabados de las cosas y por otro, sus estados iniciales y finales. Los cambios naturales tienden al estado más perfecto o acabado [teleion] de las cosas, y por ello explican los procesos retrospectivamente, es decir que el estado inicial sólo es inteligible si consideramos que ya tenía en sí mismo, en potencia, como su condición de ser, la posibilidad de dirigirse al estado final. En la definición del movimiento hay que atender a la especial relación que se da entre potencia y actualización: la clave es el en tanto que [toiouton]. Pues, el modo potencial en que se da la actualización implica no acabamiento o proceso. No obstante, el proceso será simplemente aquello que queda entre los estados inicial y final y cuya explicación se da en virtud de ellos. Porque puedo observar y reconocer el estado inicial y el final, puedo asumir que hubo cambio entre ellos. La composición del movimiento que efectúa Aristóteles, y con ello la determinación de su ontología, se erige sobre la distinción entre dynamis y entelecheia. A partir de la distinción de estos elementos se propone una jerarquía que permite hacer depender unos estados de otros y determinar así su inteligibilidad, es decir, darles el orden de la causalidad explicativa. En últimas, son conceptos que pertenecen a los cimientos de la construcción aristotélica.

El origen de la distinción entre estos dos términos se enmarca en una discusión con los megáricos. El blanco de ataque de Aristóteles es una concepción unívoca de ser que lo opone únicamente a no ser, para los megáricos solo hay poder hacer [dynasthai] cuando hay hacer o se está en acción [hotan energei]. La afirmación es radical: si no se está en acción o en proceso, no es pensable que algo esté en capacidad de hacerse o poder hacer ${ }^{12}$. Más allá de lo que los megáricos hayan dicho, la objeción de Aristóteles es

${ }^{11}$ La esencia [to ti en einai] de cada cosa determinará cuál es el estado más perfecto en cada caso.

${ }^{12}$ Hotan de me energei, ou dynasthai (Met. 1046b30). 
clara: negar la existencia de la capacidad o potencia, independientemente de la acción en curso, tiene consecuencias graves respecto del modo en que comprendemos los procesos. La acción no se reduce, al menos teóricamente, a su proceso actual de desarrollo, sino que además implica un marco de posibilidad, a saber, que para poder llegar a ser debía tener la posibilidad de ser, bajo ciertas condiciones. Este marco de posibilidad, este potencial de ser, tiene la característica de estar predeterminado por el estado acabado de lo que cambia, tiende teleológicamente a su estado más perfecto. En esto consiste el especial orden que le da Aristóteles al movimiento: compone cosas que se mueven y estados acabados dentro de un marco de posibilidad que se subordina a dichos estados acabados. Rehace el movimiento a través de unas detenciones que efectúa, unos estados sólidos y estáticos que privilegia como más inteligibles y perfectos, de manera que puede erigirlos como la causa explicativa del movimiento. Así la materia del cosmos cambiante adquiere una forma inteligible a través de unos procesos de delimitación de conceptos, de jerarquías y privilegios, bajo la directriz de una idea de orden y de relaciones causales como principios de organización. Sobre esta primera construcción se erige el tiempo.

\subsection{Tiempo}

Vosotros no podéis reconstruir el movimiento con posiciones en el espacio, o con instantes en el tiempo, es decir, con cortes [coupe] inmóviles... sólo cumplís esa reconstrucción uniendo a las posiciones o a los instantes la idea abstracta de una sucesión, de un tiempo mecánico, homogéneo, universal y calcado en el espacio (...) por más que acerquéis dos instantes o dos posiciones, el movimiento se efectuará siempre en el intervalo entre los dos, y por tanto a vuestras espaldas. (Deleuze, 1984, pp. 13-14)

La construcción aristotélica, si bien reconoce que el movimiento se da en el intervalo, pone el énfasis en los cortes inmóviles, en los instantes privilegiados. De esto resulta un compuesto abstracto: el tiempo como número del movimiento según el antes y el después (Fís. 219b). El tiempo se compone como se compone el número, se hacen análogos, se sustituyen con el fin de explicar una idea que rige el proceso de composición del tiempo: la sucesión. La sucesión consiste en que el estado inicial del movimiento va antes que su culminación que es, en relación con ella, posterior. Antes y después son conceptos que manifiestan el orden dado al movimiento, su especial composición. Así, la sucesión es la estructura temporal que se erige sobre el movimiento y que va a ser determinada bajo la forma del número. 
Conocemos también el tiempo cuando al determinar [horisomen] el antes y el después, determinamos el movimiento; y, cuando tenemos la percepción del antes y el después en el movimiento, decimos entonces que el tiempo ha transcurrido. Y lo distinguimos [horisomen] al captar que son diferentes entre sí y que hay algo intermedio diferente de ellos. Porque cuando inteligimos los extremos como diferentes del medio, el alma dice que los ahoras son dos, uno antes y otro después, es entonces cuando decimos que hay tiempo, ya que se piensa que el tiempo es lo determinado [horizomenon] por el ahora; y aceptamos esto. (Fis. 219a21-30)

Lo anterior [proteron] y lo posterior [hysteron $]^{13}$ temporales son diferentes en virtud de la diferencia en el movimiento. Ahora, la diferencia presente en el movimiento no es otra que la diferencia entre el estado inicial y el estado final, entre los cuales ocurre el movimiento. Por eso decimos que el tiempo ha pasado cuando notamos esa diferencia, cuando distinguimos los extremos como diferentes del medio. Entonces, esos extremos diferentes, como los puntos que delimitan un segmento lineal, son dos: se determinan numéricamente. Los extremos, los puntos indivisibles, corresponden en el nivel temporal a los ahoras que mantienen una relación con el tiempo análoga a la que mantienen los puntos con la línea: los ahoras no son temporales, pero limitan los intervalos temporales, así como los puntos sin longitud delimitan la línea. El tiempo se compone por la sucesión de un primer ahora, un intervalo temporal y un segundo ahora. Esta relación numérica que constituye el tiempo abstrae un aspecto de las cosas que se mueven y sus estados y procesos: la diferencia. Así el orden de sucesión de los momentos diferentes en el movimiento se torna una relación homogénea entre unidades.

La actividad de delimitar o determinar los límites entre un intervalo temporal tiene como condición la diferencia entre dos ahoras que marcan dicho intervalo. Estos ahoras respecto de la sucesión temporal funcionan como los puntos que marcan los segmentos de la línea, recordemos que esta analogía le sirve a Aristóteles para explicar la estructura continua del

\footnotetext{
${ }^{13}$ Estos términos suelen traducirse por "antes" y "después". No obstante, debe notarse que propiamente corresponden a "lo anterior" y "lo posterior" en la medida en que corresponden a adjetivos sustantivados neutros y no, adverbios de tiempo. En lo que sigue, respetaré el uso de "antes y "después" en las citas dada la traducción que estoy manejando, pero en el cuerpo del texto preferiré usar "lo anterior" y "lo posterior".
} 
movimiento. Hay una relación de dependencia entre la idea de movimiento, concebida a partir del privilegio de estados puntuales por encima de los intervalo de movimiento, y la idea de tiempo que se ordena sobre la base de un continuo de ahoras que delimitan intervalos temporales. La razón es que la diferencia entre lo anterior y lo posterior, que en el movimiento y la magnitud se determina posicionalmente, en el tiempo corresponde a la diferencia entre dos ahoras que marcan un intervalo temporal ${ }^{14}$.

En suma, el hecho de que lo anterior y lo posterior sean diferentes implica que hay dos ahoras; que sean dos, y no uno, se funda en que los ahoras son diferentes ${ }^{15}$. Por ello se entiende que Aristóteles hable del tiempo como número, podemos contar o numerar los ahoras o instantes en su diferencia. "Cuando percibimos el ahora como una unidad [hos hen] (...) o como el mismo respecto de lo anterior y lo posterior, entonces no parece que haya transcurrido algún tiempo"16 (Fís. 219a31-33). Así, considerar dos ahoras como anteriores y posteriores en el movimiento implica el tiempo. En otras palabras, cuando distinguimos lo anterior y lo posterior se hace evidente el tiempo; justamente esto significa que el ahora determina el tiempo (Fís. 219a29). Si para que haya tiempo debe haber diferencia entre el tiempo anterior y el posterior, se requiere que haya algo que marque dicha diferencia, un límite. Para que el ahora pueda cumplir dicha función de

\footnotetext{
${ }^{14}$ Goldschmidt (1982, p. 33) y Coope (2005, p. 50) defienden que hay un acompañamiento de determinaciones entre el tiempo, el movimiento y la magnitud. Ambos se oponen a la afirmación de una dependencia ontológica entre ellos. La prioridad del movimiento sobre el tiempo se da en términos explicativos para Coope, y se refiere particularmente a la relación entre tiempos y movimientos concretos, y no el tiempo y el movimiento en general. Pero, si la relación de acompañar es necesaria ¿por qué no hablar de dependencia en términos ontológicos? ¿hay algún temor a esta clase de compro miso? Ello no implica una reducción del tiempo al movimiento, sino el reconocimiento de que Aristóteles determina dicho vínculo como necesario e inherente a la definición de cada uno.

${ }^{15}$ Hay que aclarar que anterior y posterior son términos que se fijan de una manera móvil, a veces respecto del tiempo y a veces respecto de los ahoras (Fís. 219a27; 219a33; 220a11; 220b6; 220b9). Esto ha ocasionado confusión en el momento de distinguir los sentidos de ahoras. Razón por la cual hay que precisar en cada caso a qué se refieren, y en virtud de qué pregunta o problema se hizo esa asignación, por decirlo así.

${ }^{16}$ Sugiero traducir la expresión hos hen por "como uno", pues lo que está en juego es la diferencia que aparece cuando se consideran dos ahoras, no uno. La confusión que se genera aquí es grande pues corremos el riesgo de pensar que el ahora es la unidad del tiempo. En esto me alejo de la interpretación de Julia Annas (1975, pp. 109-110).
}

UNIVERSITAS PHILOSOPHICA, 31(63), pp. 267-286 - ISSN 0120-5323 
marcar o delimitar los intervalos temporales este debe ser indivisible ${ }^{17} . \mathrm{Si}$ el ahora pudiera dividirse tendría una magnitud temporal y por ello sería un segmento del tiempo y no el límite del mismo. De ahí que la analogía de la línea y el punto esté vigente también en el nivel temporal. Así pues, el ahora en tanto límite debe tener dos funciones: por un lado, mantener unidas las partes del tiempo, y por otro, dividirlas, esto es, marcar su diferencia.

Construir el concepto de tiempo significa, entonces, darle el orden de la sucesión al movimiento. Pero el movimiento no solo tiene un orden temporal, sino que él mismo también está ordenado: el tránsito o cambio de un estado a otro se explica o se ordena según los fines hacia los cuales tienden las cosas en un proceso de perfeccionamiento. Entonces el tiempo ordena los ahoras sucesivos y los delimita en correspondencia con los estados acabados de las cosas que comienzan y terminan en el tiempo: alcanzar el estado de perfección toma tiempo y las cosas que cambian son temporales en esa medida. El concepto de tiempo de Aristóteles depende fundamentalmente del concepto teleológico del movimiento; la analogía del punto y la línea me permitió explicar esto, en cuanto permite notar cómo la estructura continua del movimiento se superpone y entrelaza a la estructura continua del tiempo.

\section{Conclusiones}

HE CONSIDERADO EL TIEMPO Y EL MOVIMIENTO COMO CONCEPTOS desde el punto de vista de su construcción. Así como la concha artificial del artesano en Valéry,

\footnotetext{
${ }^{17}$ Hay comentaristas que sostienen un sentido del ahora como persistente y por ello como un periodo de tiempo, de lo cual resulta que es divisible, para ello pueden verse las notas de Bostock en la traducción de Waterfield de la Física (2008, 264-65). El argumento se inscribe dentro de una interpretación de la relación del pheromenon, lo que se mueve, con el ahora (Fís. 220a1-4) que establece una analogía entre la permanencia en el cambio del primero y una permanencia del ahora en el tiempo. Esto genera una confusión mayor en el momento en que se dice que el ahora es la unidad del tiempo, lo cual es incongruente con su función de límite (Annas, 1975, pp. 109-110). La hipótesis es que así como el movimiento es el desplazamiento de algo, una línea es el desplazamiento, si bien no posicional, de un punto. Pero creer que una línea es el desplazamiento de un punto es problemático, pues una línea no se compone de puntos, ni de uno, ni de muchos (Fís. 220a20; 239b5ss) No obstante, el problema de la indivisibilidad se mantiene ipuede algo indivisible estar en movimiento? "Todo lo que cambia tiene que ser divisible" (Fís. 234b10). Dentro del concepto de movimiento aristotélico no cabe la posibilidad de que un punto se mueva. Por tanto, tampoco el ahora en su función de límite de los intervalos temporales puede "estar en movimiento" o durar en el tiempo, que sería el equivalente al movimiento en el nivel temporal de la estructura del continuo.
} 
Aristóteles tuvo que tomar decisiones en cuanto a la "forma", en este caso conceptual, del tiempo y del movimiento. Dicha acción de dar forma consiste en una manera de ordenar y escoger ciertos elementos de manera que se establezcan relaciones explicativas entre ellos, las cuales están dadas a partir de privilegios y jerarquías ontológicas y causales; tales como el privilegio de la actualización sobre la potencialidad, y la determinación de la primera como más perfecta respecto del proceso que toma alcanzarla teleológicamente. A esto he llamado una construcción conceptual, particularmente del movimiento. También mostré cómo la analogía con el punto y la línea encaja no sólo con la peculiar relación entre la potencialidad y la actualidad, sino además con la estructura de sucesión numérica que Aristóteles le atribuye al tiempo. Entonces, sobre la base de la analogía con los segmentos lineales y los puntos que los dividen se construye la estructura del tiempo en términos de segmentos temporales, divididos por ahoras que cumplen la función de límites de dichos intervalos o segmentos. En este contexto adquiere sentido la dependencia que establece el estagirita entre el tiempo y el movimiento, en tanto que son conceptos construidos uno de la mano del otro, sólo si comprendemos el modelo de explicación, o de producción, del movimiento podemos entender aquel propio del tiempo.

La distinción entre la producción humana (construcción) y la producción (natural), para la cual utilicé las referencias a Paul Valéry, me permitió notar cómo la construcción de un concepto con el fin de hacer inteligible un fenómeno es más parecida a la producción del artesano, y a su condición humana, que a la producción natural. En ocasiones tratamos los conceptos como seres de la naturaleza hasta el punto en que perdemos de vista que su factura es humana. A propósito de la búsqueda de la verdad, solemos conformarnos con afirmar que un concepto tiene sentido dentro de un marco explicativo del mundo y perdemos de vista muy frecuentemente que el sentido del concepto depende del modo como ha sido construido dicho marco. Al notar las decisiones implícitas en la construcción de un concepto es posible comprenderlo mejor, pues podemos recomponer el proceso de construcción del mismo o rehacerlo con el pensamiento. Cuando Aristóteles inscribe el concepto de tiempo en la naturaleza pierde de vista los efectos de su labor filosófica sobre el concepto. Por esta razón, el tiempo no es algo natural sin más; una aproximación al concepto de tiempo aristotélico debería tener en cuenta que este depende del modo particular en que el autor lo entrelaza con la ontología del movimiento y ella, a su vez, con una visión determinada de la 
physis. En este sentido "poiesis del tiempo y el movimiento" hace referencia a la construcción humana, aristotélica, del concepto de tiempo en relación con el concepto de movimiento.

\section{Referencias}

Annas, J. (1975). Aristotle, Number and Time. The Philosophical Quarterly, 25(99), pp. 97-113.

Aristóteles. (2008). Physics. (Trad. R. Waterfield, introducción y notas de D. Bostock). New York: Oxford University Press.

Aristóteles. (1995). Física. (Trad. G. de Echandía). Madrid: Gredos.

Aristóteles. (1984). The Complete Works of Aristotle. The Revised Oxford Translation. Barnes J. (Ed.). Princeton: Princeton University Press.

Aristóteles. (1977). Metafísica de Aristóteles. (Trad. V. García Yebra). Madrid: Gredos.

Bostocк, D. (2006). Space, Matter and Form: Essays on Aristotle's Physics. New York: Oxfod University Press.

Brigante, A. M. (2008). Obstinado rigor. La teoría de la acción poética de Paul Valéry. Bogotá: Editorial Pontifica Universidad Javeriana.

Coope, U. (2005). Time for Aristotle: Physics IV.10-14. New York: Oxford University Press.

Deleuze, G. (1984). La imagen-movimiento: Estudios sobre cine I. Barcelona: Paidós.

Gambra, J. M. (1996). El número en Aristóteles. Thémata, 17, pp. 45-74

Hussey, E. (1993). Aristotle's Physics Books III and I. New York: Oxford University Press.

Johnson, M. R. (2005). Aristotle on Teleology. Oxford: Oxford University Press.

Ross, W.D. (1936). Aristotle's Physics. A Revised Text with Introduction and Commentary. Oxford: Clarendon Press.

Valéry, P. (1998). Primera lección del curso de Poética. Teoría poética y estética (pp. 105-129). Madrid: Visor. 
ValÉry, P. (1993). El hombre y la concha. Estudios Filosóficos (pp. 139-162). Madrid: Visor.

Waterlow, S. (1984a). Aristotle's Now. The Philosophical Quarterly, 34(135), pp. 104-128.

Waterlow, S. (1984b). Aristotle's Physics Books III and IV by Edward Hussey. The British Journal of the Philosophy of Science, 35(4), pp. 404-408.

Wieland, W. (1992). Die aristotelische Physik. Göttingen: Vandenhoek \& Ruprecht. 